\title{
Pulse front tilt steering in laser plasma accelerators
}

\author{
Maxence Thévenet, ${ }^{*}$ Daniel E. Mittelberger, ${ }^{\dagger}$ Kei Nakamura, Rémi Lehe, Carl B. Schroeder, \\ Jean-Luc Vay, Eric Esarey, and Wim P. Leemans \\ Lawrence Berkeley National Laboratory, Berkeley, California 94720, USA
}

(Received 23 April 2018; published 15 July 2019)

\begin{abstract}
We report on the effect of laser spatiotemporal coupling in laser plasma accelerators. Pulse front tilt in the driving laser causes asymmetry in the wakefield, resulting in deflection of the electron beam from the laser axis. We explore the physical mechanisms and propose a quantitative model of electron steering, which is validated with particle-in-cell simulations. Even a small amount of pulse front tilt can result in beam steering in the final down ramp of the plasma profile, which may lead to unexpected beam-pointing errors or fluctuations. On the other hand, it can be used to govern the final beam direction, which has consequences for staging laser plasma accelerators in a high-energy physics collider as well as $\mathrm{x}$-ray generation for biological imaging.
\end{abstract}

DOI: 10.1103/PhysRevAccelBeams.22.071301

In the past two decades, laser plasma accelerators (LPAs) have produced electron beams with increasing quality and energy. The plasma waves generated by a high-intensity laser pulse can sustain accelerating gradients of tens of $\mathrm{GV} / \mathrm{m}[1]$, thus surpassing the electric field achievable with conventional radio-frequency-based technology by orders of magnitude and allowing smaller accelerating structures [2]. The current energy record is $4.2 \mathrm{GeV}$ using a $9 \mathrm{~cm}$ accelerating structure [3].

Improving electron beam quality and understanding beam degradation is an active field of research. Defects and spatiotemporal coupling in the laser pulse were shown to modify the electron beam dynamics [4]. One particular form of spatiotemporal coupling, pulse front tilt (PFT), was observed to affect the electron trajectories in particle-in-cell (PIC) simulations and in experiments [5,6], resulting in deflection of the accelerated electrons with respect to the laser axis. Furthermore, PFT can cause large-amplitude coherent betatron oscillations, hence allowing brighter $\mathrm{x}$-ray sources based on betatron radiation used to image biological samples [7].

Electron-pointing control and stability are crucial for numerous LPA applications like high-energy physics [8], $\mathrm{x}$-ray generation [9], radiation biology [10], and the study

\footnotetext{
*mthevenet@lbl.gov

'Present address: Lawrence Livermore National Laboratory, Livermore, California 94550, USA.

Present address: Deutsches Elektronen-Synchrotron DESY, 22607 Hamburg, Germany.

Published by the American Physical Society under the terms of the Creative Commons Attribution 4.0 International license. Further distribution of this work must maintain attribution to the author(s) and the published article's title, journal citation, and DOI.
}

of quantum electro-dynamics effect $[11,12]$. As an example, an LPA-based collider [13] requires a chain of tens of consecutive LPA stages [14], and pointing fluctuations in the range of $1 \mathrm{mrad}$ at the end of an accelerator stage lead to an unacceptable offset of $1 \mathrm{~mm}$ at the entrance of the following stage $1 \mathrm{~m}$ further.

In this paper, we propose a model for electron steering due to pulse front tilt in a laser wakefield accelerator. The model is verified by comparison with PIC simulations of an LPA performed with codes WarpX [15] and Warp [16]. It provides quantitative information on the acceptable PFT fluctuations as well as a practical way to control the final electron beam direction in an LPA. Moreover, we show that, in the presence of pulse front tilt, the shape of the plasma tail can have a severe impact on the final beam pointing.

Spatiotemporal coupling of a laser pulse is the nonseparability of the space and time dependence of the fields: $f(x, t) \neq f_{x}(x) \times f_{t}(t)$, where $f$ can be the electric or the magnetic field, $x$ is a transverse coordinate, and $t$ is time. PFT is defined by linear dependence of the laser group delay on the transverse coordinate, resulting in an angle between the laser intensity front and phase fronts. It has been shown to deflect the accelerated electron beams [5] or enhance off-axis electron injection into the laser wake and partially polarize the resulting betatron $\mathrm{x}$ rays [6]. The PFT angle depends on the main spatiotemporal coupling parameters as [17]

$$
\tan \psi=c\left(k_{L} \beta+\varphi_{2} \frac{\zeta}{\zeta^{2}+\frac{w^{2} \tau^{2}}{4}}\right),
$$

where $\psi$ is the PFT angle, $c$ is the speed of light, $k_{L}$ is the laser central wave number, $w$ is the pulse transverse width, 
and $\tau$ is the bandwidth-limited pulse duration and we used the definitions of Ref. [17] for $\beta, \varphi_{2}$, and $\zeta$, the angular, temporal, and spatial chirp, respectively. This equation is valid for a Gaussian pulse, and the evolution of $\beta, \varphi_{2}$, and $\zeta$ can be altered through propagation in a plasma.

A laser pulse propagates through a plasma with electron density $n_{e}$, plasma frequency $\omega_{p} \propto \sqrt{n_{e}}$, and plasma wave number $k_{p}=\omega_{p} / c$. We assume the resonant condition $\omega_{p} \tau \sim 1$ is satisfied, and we use $a_{0}=e A / m_{e} c^{2}$ for the normalized laser vector potential, with $A$ the amplitude of the laser vector potential and $e$ and $m_{e}$ the elementary charge and electron mass, respectively. The laser drives plasma density waves in its wake, generating large longitudinal and transverse fields that can accelerate and focus charged particles. We hereafter demonstrate how PFT in the driving laser tilts the wake structure and how the evolution of this tilt results in electron beam steering.

Let $z$ be the laser propagation direction and $\xi=z-v_{g} t$ the copropagating variable with $v_{g}$ the laser group velocity. The laser pulse center on axis is located at $\xi=0$. Spatiotemporal coupling is introduced along the transverse direction $x$, and the electron dynamics are analyzed in the $x-z$ plane. We assume that the laser pulse is linearly polarized and is Gaussian in time and space. The laser transverse vector potential envelope is defined by $a_{L}(x, \xi)=a_{0} \exp \left[-\frac{(\xi+x \tan \psi)^{2}}{(c \tau)^{2}}\right] \exp \left[-\frac{x^{2}}{w^{2}}\right]$, where $w$ is the pulse spatial width and $(x / c) \tan \psi$ is the center of the temporal envelope, or laser group delay, at position $x$. The beam waist, i.e., its transverse width at focus, is denoted by $w_{0}$ and is associated to the Rayleigh length $z_{R}$ through $z_{R}=\pi w_{0}^{2} / \lambda_{L}$, where $\lambda_{L}$ is the laser central wavelength related to laser central frequency $\omega_{L}$ by $\omega_{L}=2 \pi c / \lambda_{L}$. In the rest of this article, $\lambda_{L}=0.8 \mu \mathrm{m}$.

All PIC simulations were performed with the Boris pusher, finite-difference time domain Cole-Karkkainen solver [18] with Cowan coefficients [19] and third-order shape factor for current deposition and field gather. The time step is set to the Courant condition for the ColeKarkkainen field solver $\Delta t=\Delta z / c$. A filter was applied to mitigate numerical Cherenkov instability [20]. All boundaries have an absorbing condition. Simulations were performed in a boosted frame [21]. We used the field expressions from Ref. [17].

In the presence of PFT, the wake is distorted and trapped electrons propagate at $x \neq 0$, as illustrated in Fig. 1 . Electrons perform betatron oscillations around the wake line, where the transverse force $F_{x}$ is zero $\left(E_{x}=0\right.$ in the linear regime). The simulation was performed with the PIC code Warp [16,22]. The 2D $x-z$ simulation box is $500 \mu \mathrm{m} \times 120 \mu \mathrm{m}$ wide with $462 \times 4629$ cells, with one particle per cell.

We hereafter derive an equation for this conditions. In order to calculate the wake profile, the following assumptions are made: (i) The interaction takes place in the linear or quasilinear regime: $a_{0} \lesssim 1$; (ii) the PFT angle is small:

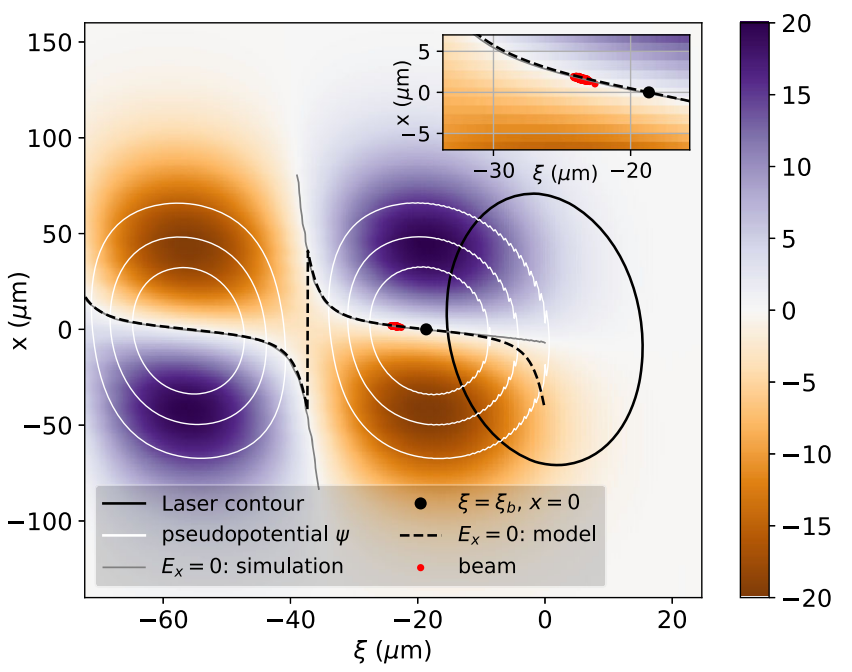

FIG. 1. Transverse electric field $(\mathrm{GV} / \mathrm{m})$ in a 2D LPA simulation with a $\tau=60 \mathrm{fs}, w_{0}=70 \mu \mathrm{m} y$-polarized laser pulse with $a_{0}=0.1$ propagating in a plasma with density $2 \times 10^{17} \mathrm{~cm}^{-3}$. The PFT angle $\psi \simeq 30 \mathrm{mrad}$ at the snapshot location is due to $\beta=0, \varphi_{2}=500 \mathrm{fs}^{2}$, and $\zeta=0.5 \mathrm{~mm} \mathrm{fs}$ at focus, resulting in an $8.5 \%$ intensity drop. The black solid-line ellipse is an isocontour of $1 / 4$ laser intensity. A short electron beam (red) is at equilibrium off axis, as can be seen in the enlargement (inset).

$k_{p} w \tan \psi \ll 1$. In the linear regime, the pseudopotential $\Psi$, defined by $\Psi=\phi-a_{z}$ with $\phi$ the normalized electrostatic potential and $a_{z}$ the longitudinal vector potential, can be obtained through integration along the $\xi$ coordinate only [23]. It reads $\Psi=\Psi_{0} \sin \left[k_{p}(\xi-x \tan \psi)\right] \exp \left(-2 x^{2} / w^{2}\right)$ behind the laser, where $\Psi_{0}$ depends on the pulse intensity and shape. This equation shows that the plasma phase delay equals the laser group delay for any $x$ in the quasistatic linear regime. The wake line, defined by $F_{x}=e \partial \Psi / \partial x=0$, is given by

$$
\tan \psi+\frac{4 x}{k_{p} w^{2}} \tan \left[k_{p}(\xi-x \tan \psi)\right]=0 .
$$

This expression was used to plot the dashed line in Fig. 1, which shows excellent agreement with the simulation (gray line). As this equation cannot be solved analytically to get $x=f(\xi)$, we propose a simplified model. A Taylor expansion close to the center of the first bucket $\xi_{b}=-(\pi / 2) k_{p}^{-1}$ (where the wake line intersects the $z$ axis, black dot in Fig. 1) gives the tilt $\theta_{w}$ of the wake line:

$$
\tan \theta_{w}=-\left(\frac{k_{p} w}{2}\right)^{2} \tan \psi .
$$

An electron located at $\xi=\xi_{e}$ behind the laser pulse, with transverse position $x_{e}$, performs betatron oscillations according to $\ddot{x}_{e}+c^{2} k_{\beta}^{2}\left(x_{e}-x_{E q}\right)=0$, where $k_{\beta}$ is the betatron wave number and $x_{E q}$ is the equilibrium position given by 


$$
x_{E q}=\left(\xi_{b}-\xi_{e}\right) \tan \theta_{w} .
$$

In the adiabatic regime, where all quantities evolve slowly with respect to the betatron frequency, the beam position equals the equilibrium position $x_{E q}$ at all time. We hereafter present general considerations on the adiabatic regime and then explore the effect of the final plasma down ramp, where the condition of adiabaticity breaks.

Pulse front tilt results in electron steering in the adiabatic regime if at least one of these conditions is met: (i) The laser properties evolve ( $\psi, w \neq$ const); (ii) the density is not constant $\left(k_{p} \neq\right.$ const); or (iii) there is significant electron dephasing $\left(\xi_{e} \neq\right.$ const). To clarify the mechanisms, we present a concrete example of case (i), which we refer to as dynamic steering, and of case (ii), which we refer to as down ramp steering, in the adiabatic regime [case (iii) is somehow uncommon]. In principle, pulse front tilt can also result in laser steering, where the transverse position of the laser pulse centroid evolves [24]. In the rest of this study, this effect is negligible.

Dynamic steering occurs when the laser properties evolve along propagation: $\psi, w \neq$ const. This is typically the case in a uniform plasma that is longer than the laser Rayleigh length. Figure 2(a) shows a comparison between electron trajectories from a numerical simulation and the model, where the Rayleigh length $(2.5 \mathrm{~mm})$ is much smaller than the plasma length $(20 \mathrm{~mm})$. The simulations are performed with the PIC code WarpX, and the box is $800 \mu \mathrm{m} \times 90 \mu \mathrm{m}$ wide with $493 \times 2777$ cells, with one particle per cell. The accelerated electrons, plotted in gray, perform betatron oscillations around the transverse equilibrium position. Here, the PFT decreases on average, so electrons located behind the bucket center are steered upward, in agreement with Eqs. (3) and (4).

Down ramp steering occurs when the density changes along propagation: $k_{p} \neq$ const. Figure 2(b) shows results from a PIC simulation compared with the theory for transverse equilibrium position. The electron bunch starts with its centroid on the wake line $\left(x_{e}=10 \mu \mathrm{m}\right)$ and is steered downward as the density drops and the bucket expands. A similar simulation with constant density shows a constant beam transverse position at $x_{e} \simeq 10 \mu \mathrm{m}$. Both cases show good agreement between PIC simulations and theory. In all cases, the final electron energy depends very weakly on the presence of PFT.

When the laser propagation is little affected by the plasma (in particular, when the laser power $P$ verifies $P<P_{c}$, where $P_{c}$ is the critical power [2]), the equation for the transverse equilibrium position $x_{E q}$ along propagation can be derived analytically for a Gaussian pulse focused at $z=z_{f}$. In the presence of spatial chirp $\zeta$ at focus, the PFT reads $\tan \psi(z)=\omega_{L} \zeta\left(z-z_{f}\right) /\left[\left(z-z_{f}\right)^{2}+z_{R}^{2}\right]$ (see, e.g., Ref. [25]). Using Eqs. (3) and (4), the expression for the transverse equilibrium position reads
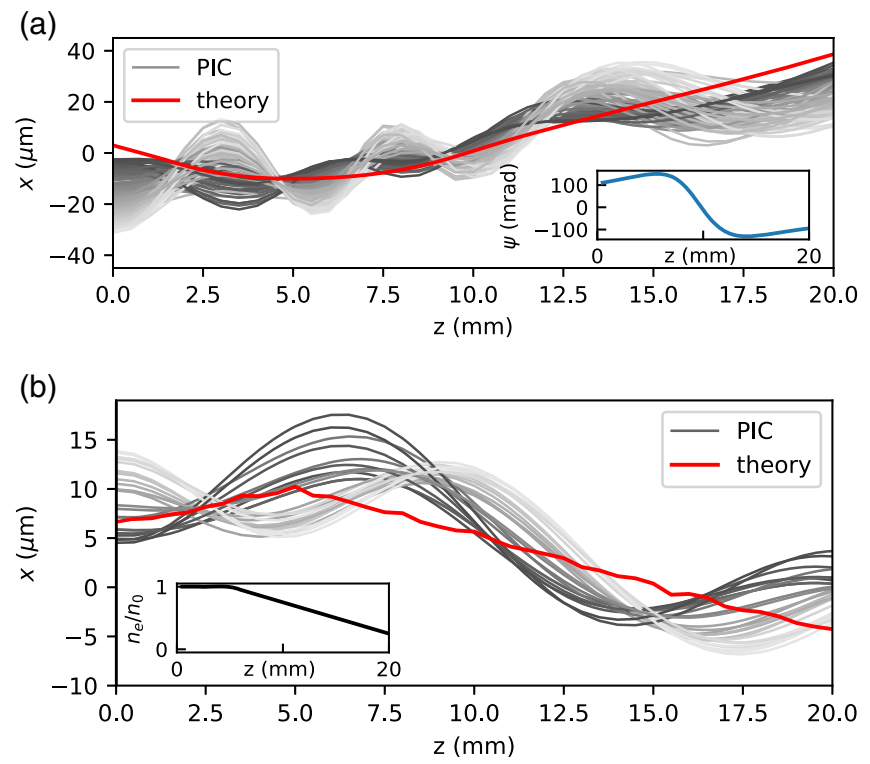

FIG. 2. (a) Electrons transverse position along propagation from a 2D PIC simulation (gray) and theory for transverse equilibrium position (red line) for dynamic steering: The plasma density is constant at $n_{e}=3 \times 10^{17} \mathrm{~cm}^{-3}$. The 24 fs $y$-polarized laser pulse is focused to a $20 \mu \mathrm{m}$ waist at $z=10 \mathrm{~mm}$ with $a_{0}=1.3$, where quasilinear approximation roughly holds. The subset shows the PFT angle evolution due to $\beta=0 \mathrm{~s}$, $\zeta=-0.5 \mathrm{~mm}$ fs, and $\varphi_{2}=500 \mathrm{fs}^{2}$ at focus, leading to a $50 \%$ intensity drop. The initial electron energy is $10 \mathrm{MeV}$. The discrepancy for $z<2.5 \mathrm{~mm}$ is due to the beam injection at $z=0 \mathrm{~mm}$. (b) The same quantities plotted for down ramp steering: The density drops linearly from 5 to $2 \times 10^{16} \mathrm{~cm}^{-3}$ between $z=5 \mathrm{~mm}$ and $z=20 \mathrm{~mm}$. The laser pulse with $w_{0}=$ $180 \mu \mathrm{m}$ and $a_{0}=0.5$ is collimated, with a constant PFT angle $\psi \simeq 64 \mathrm{mrad}$ due to $\tau=150 \mathrm{fs}, \beta=0.02 \mathrm{fs}, \zeta=0 \mathrm{fsm}$, and $\varphi_{2}=100 \mathrm{fs}^{2}$ at focus. The initial electron energy is $5 \mathrm{MeV}$. The intensity drop is $1.2 \%$.

$$
x_{E q}(z)=\left(\frac{\pi}{2 k_{p}}+\xi_{e}\right)\left(\frac{k_{p} w_{0}}{2}\right)^{2} \omega_{L} \zeta \frac{z-z_{f}}{z_{R}^{2}} .
$$

In a homogeneous plasma $\left(k_{p}=\right.$ const $), x_{E q}$ increases linearly with $z$ when $\zeta>0$ due to laser pulse diffraction. In the tail of the plasma profile, $k_{p} \rightarrow 0$ as the density drops to zero, so that steering always occurs in the final down ramp, leading to beam deflection. The rest of this article investigates this effect.

We hereafter present numerical results and general considerations on the steep ramp regime $\left(d n_{e} / d z \gg n_{e} / \lambda_{\beta}\right)$ with $\lambda_{\beta}$ the betatron wavelength, where the hypothesis of adiabaticity breaks and electrons receive a transverse kick in the down ramp. The transition to the smooth ramp regime $\left(d n_{e} / d z \ll n_{e} / \lambda_{\beta}\right)$, where the condition of adiabaticity holds and the beam follows $x_{E q}(z)$ [see Fig. 2(b)], is considered thereafter. In practice, the betatron wavelength increases $\left(\lambda_{\beta} \rightarrow \infty\right)$ as the density goes to 0 at the end of the plasma down ramp. 
(a)

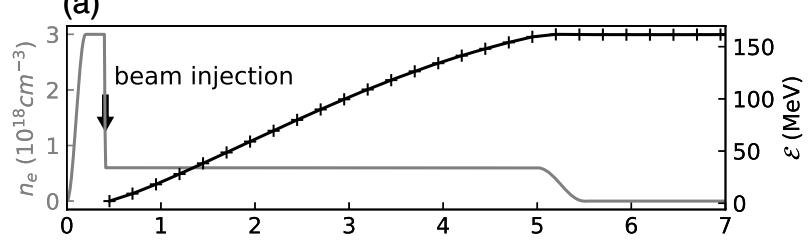

(b)

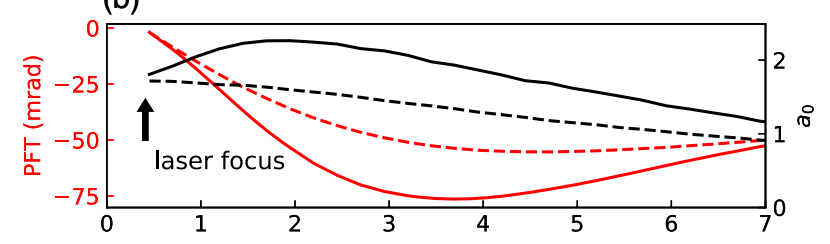

(c)

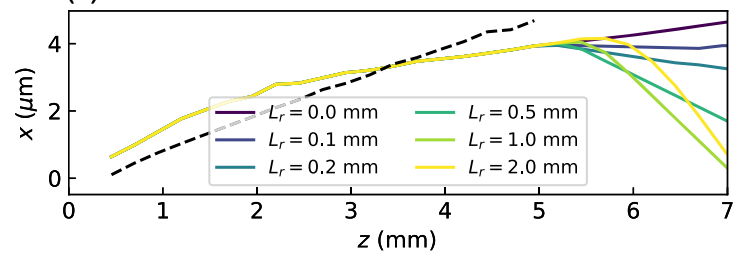

FIG. 3. (a) Plasma density profile and energy of the electron beam trapped at $z \simeq 400 \mu \mathrm{m}$. (b) Evolution of laser properties. The $\tau=20 \mathrm{fs}$ pulse is focused to $w_{0}=30 \mu \mathrm{m}$ at the end of the down ramp $z_{f}=410 \mu \mathrm{m}$, reaching $a_{0}=1.77$. Pulse front tilt is introduced with angular chirp only, with $\zeta=0.2 \mathrm{~mm}$ fs. Dashed (solid) lines show vacuum (plasma) propagation. (c) Transverse position of the electron beam upon propagation, for various lengths of the final down ramp. The dashed line shows model predictions for $x_{E q}$ [Eq. (4)].

A scan of 3D PIC simulations was performed using the code WarpX in the steep ramp regime, achieved in a majority of LPA experiments. A $2 \mathrm{~J}$ laser pulse propagates through a $4.59 \mathrm{~mm}$ plasma jet with density $n_{e}=6 \times 10^{17} \mathrm{~cm}^{-3}$ preceded with a $200 \mu \mathrm{m}$ high-density plateau $\left(n_{e}=\right.$ $3 \times 10^{18} \mathrm{~cm}^{-3}$ ) at the end of which the electron beam is injected through down ramp injection.

The plasma profile contains three cosine-shaped ramps $n_{e}(z) \propto 0.5\left[1+\cos \left(\pi z / L_{\text {ramp }}\right)\right]$ with lengths $200 \mu \mathrm{m}$, $10 \mu \mathrm{m}$ (for injection), and $0 \rightarrow 2 \mathrm{~mm}$, respectively. The simulation box is $320 \mu \mathrm{m} \times 320 \mu \mathrm{m} \times 80 \mu \mathrm{m}$ wide with $384 \times 384 \times 3072$ grid points, and we used eight particles per cell. Convergence tests show that this resolution is high enough to resolve the laser evolution and the pointing of the electron beam (see the Appendix). The simulations ran in a boosted frame with Lorentz factor $\gamma_{\text {boost }}=6$.

Figure 3(a) shows the plasma profile and the energy of trapped electrons along propagation. The laser evolution, shown in Fig. 3(b), differs significantly from vacuum propagation: The beam is self-focused around $z=2 \mathrm{~mm}$, and the PFT is consistently higher in the plasma than it is in vacuum. Because of dynamic steering in the low-density plateau $(z>4.01 \mathrm{~mm})$, the beam transverse offset reaches $x_{e}=4 \mu \mathrm{m}$, as shown in Fig. 3(c). Our model (dashed line) shows good agreement with the simulations in spite of the

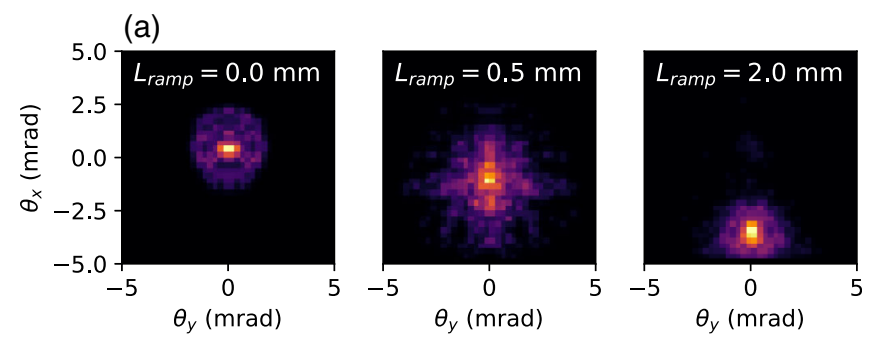

(b)

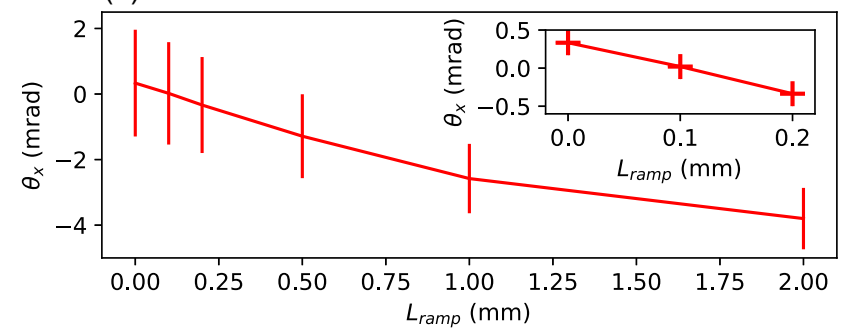

(c)

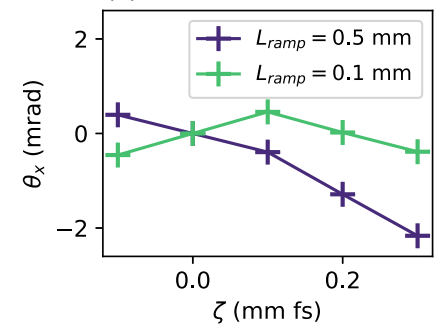

(d)

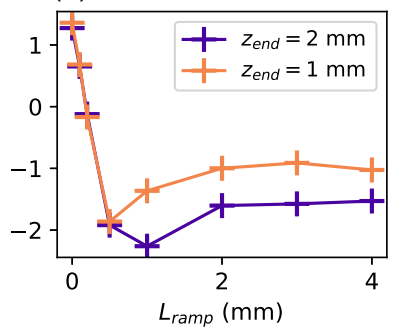

FIG. 4. (a) Final angular profile of the accelerated electron beam, for three values of the down ramp length. (b) Beam final direction as a function of the ramp length. Vertical bars show the beam width (standard deviation) in $x$. The inset is an enlargement of the first three points, without error bars. (c) Final beam angle as a function of spatial chirp at focus $\zeta$ for correcting $\left(L_{\text {ramp }}=0.1 \mathrm{~mm}\right)$ and noncorrecting $\left(L_{\text {ramp }}=0.5 \mathrm{~mm}\right)$ lengths. (d) Final beam angle as a function of ramp length $L_{\text {ramp }}$. For this subplot only, the low-density plateau was shortened (see the main text).

nonlinear regime, beam loading, and laser pulse evolution. In the absence of a down ramp (darkest purple line), the electron beam escapes with a positive angle $\theta_{x} \simeq$ $0.2 \mathrm{mrad}>0$ due to dynamic steering. In the presence of a steep down ramp $\left(L_{\text {ramp }}<\lambda_{\beta} \simeq 5 \mathrm{~mm}\right)$, the equilibrium position suddenly goes from $x=4 \mu \mathrm{m}$ to $x=0 \mu \mathrm{m}$ [see Eqs. (3) and (4)]. The electrons do not have time to adapt adiabatically and instead perform a fraction of a betatron oscillation, so the beam receives a kick.

Figures 4(a)-4(c) show the final beam distribution as a function of the final down ramp length in the steep ramp regime. As can be seen in Fig. 4(a), the beam deflection angle can be much larger than the beam divergence. Figure 4(b) shows the final beam direction as a function of the down ramp length. Since dynamic steering leads to $\theta_{x}>0$ and down ramp steering kicks the beam downward, there exists a correcting length $\left(L_{\mathrm{ramp}} \simeq 0.1 \mathrm{~mm}\right.$ in this scan) for which down ramp steering exactly compensates dynamic steering, as shown in the inset in Fig. 4(b). As can 
be seen in Fig. 4(c), choosing the correcting length helps keep the beam deflection below $0.4 \mathrm{mrad}$ for a wide range of $\zeta$, while it reaches $2 \mathrm{mrad}$ for a noncorrecting ramp length. Better control on the final direction can be achieved by fine tailoring of the ramp, as done in Ref. [26] for emittance conservation. As can be seen in Fig. 4(b), the down ramp length also has an influence on beam divergence.

Similarly to what is done in Ref. [27] for emittance preservation, a smooth ramp can also compensate PFT steering. To do so, the plasma density should drop fast enough in the down ramp so that down ramp steering compensates dynamic steering. However, depending on the laser parameters (focal position and Rayleigh range), it is not always possible to compensate dynamic steering with down ramp steering and obtain a final direction for the electron beam parallel to the $z$ axis. As an illustration, Fig. 4(d) shows the final angle as a function of $L_{\text {ramp }}$ for two scans with the same parameters as previously (see the caption of Fig. 3) except that the end of the low-density plasma plateau $z_{\text {end }}$ was reduced from $5 \mathrm{~mm}$ to $z_{\text {end }}=$ $1 \mathrm{~mm}(2 \mathrm{~mm})$. For $z_{\text {end }}=1 \mathrm{~mm}(2 \mathrm{~mm})$, the betatron wavelength is $1 \mathrm{~mm}(1.5 \mathrm{~mm})$, and the beam angle plateaus at $\theta_{x}=-1 \mathrm{mrad}(-1.5 \mathrm{mrad})$ when the gradient length is increased up to $4 \mathrm{~mm}$. Even though the beam is not parallel to the $z$ axis, its final direction is not sensitive to the length of the down ramp within a few-millimeter range. This might be an appropriate regime to achieve a stable and consistent steering, which is valuable for LPA staging experiments.

In this paper, we demonstrate how pulse front tilt in the driving laser of a laser plasma accelerator affects the electron propagation and deflects the accelerated electron beam: The wake structure is tilted, and the electron beam travels off axis. A model of electron steering due to pulse front tilt is developed in the linear regime and shown to be in good agreement with particle-in-cell simulations. The results show that strict control over pulse front tilt is required to generate high-quality electron beams, needed for staging laser plasma accelerators or free electron lasers based on wakefield acceleration [9]. Even with a small amount of pulse front tilt, a significant deflection of the electron beam can occur in the plasma down ramp. This effect might be a cause of beam-pointing fluctuations in experiments where the laser pulse evolution results in pulse front tilt and could also be used to deliberately steer the electron beam to angularly separate the electron beam from the laser pulse, as can be required in staging experiments.

\section{ACKNOWLEDGEMENTS}

The authors acknowledge the contributions of Anthony Gonsalves, Carlo Benedetti, and Sven Steinke for their perspectives. This work was supported by the Director, Office of Science, of the U.S. Department of Energy (DOE) under Contract No. DE-AC02-05CH11231. This research used resources of the National Energy Research Scientific Computing Center (NERSC), a U.S. Department of Energy

Office of Science User Facility operated under Contract No. DE-AC02-5CH11231. WarpX is supported by the Exascale Computing Project (17-SC-20-SC), a collaborative effort of two U.S. Department of Energy organizations (Office of Science and the National Nuclear Security Administration).

\section{APPENDIX: NUMERICAL CONVERGENCE}

Convergence tests were performed so as to confirm the validity of the 3D study presented above. In the quasilinear regime investigated here, the resolution must be sufficient to capture the laser pulse width (transversally) and laser wavelength (longitudinally), to properly describe the laser pulse propagation, which is equivalent to $k_{L x} \Delta x \ll 1$ and $k_{L z} \Delta z \ll 1$, where $k_{L x}$ and $k_{L z}$ are the transverse and longitudinal components, respectively, of the laser wave vector and $\Delta x$ and $\Delta z$ and the transverse and longitudinal cell sizes, respectively. In this study, we used $k_{L x} \Delta x=0.015$ and $k_{L z} \Delta z=0.19$.

The 3D simulation presented in Figs. 3 and 4 for $L_{\text {ramp }}=$ $1 \mathrm{~mm}$ was compared to a run with a $1.5 \times$ higher resolution. Figure 5 shows the comparison between the original resolution (reference) and the higher resolution (high). As expected, the laser evolution is properly captured [see Figs. 5(a) and 5(b)].

While the resolution may not be sufficient to fully resolve the beam injection and beam loading precisely, we anticipate that the direction of the electron beam should not be too sensitive to these effects. As a confirmation, the beam direction is shown in Fig. 5 for the two resolutions

(a)

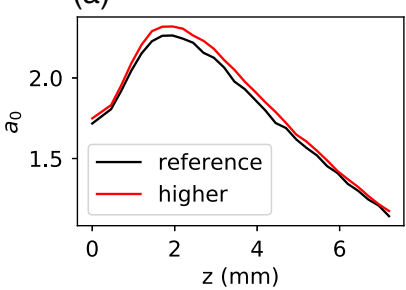

(c)

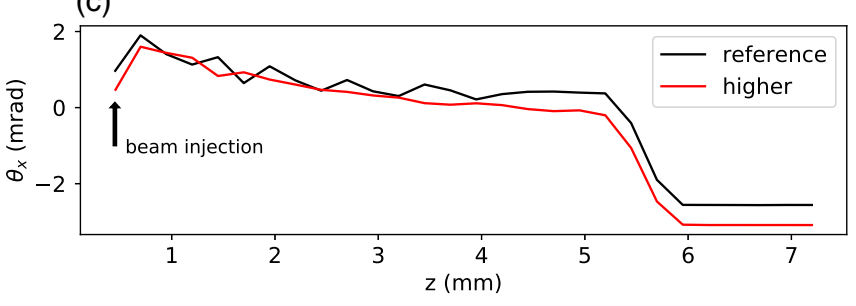

FIG. 5. Evolution of laser pulse and electron beam parameters during propagation for two simulations with the same physical parameters, with the reference resolution (transverse and longitudinal cell sizes are $0.833 \mu \mathrm{m}$ and $26.0 \mathrm{~nm}$, respectively, in black) and a higher resolution (transverse and longitudinal cell sizes are $0.625 \mu \mathrm{m}$ and $19.5 \mathrm{~nm}$, respectively, in red). (a) Laser normalized amplitude $a_{0}$; (b) laser pulse front tilt angle; (c) electron beam propagation direction $\theta_{x}$. The length of the final down ramp is $1 \mathrm{~mm}$. 
mentioned above. The beam direction shows very little dependence on the resolution.

[1] T. Tajima and J. M. Dawson, Laser Electron Accelerator, Phys. Rev. Lett. 43, 267 (1979).

[2] E. Esarey, C. Schroeder, and W. Leemans, Physics of laserdriven plasma-based electron accelerators, Rev. Mod. Phys. 81, 1229 (2009).

[3] W. Leemans, A. Gonsalves, H.-S. Mao, K. Nakamura, C. Benedetti, C. Schroeder, C. Tóth, J. Daniels, D. Mittelberger, S. Bulanov et al., Multi-GeV Electron Beams from Capillary-Discharge-Guided Subpetawatt Laser Pulses in the Self-Trapping Regime, Phys. Rev. Lett. 113, 245002 (2014).

[4] B. Beaurepaire, A. Vernier, M. Bocoum, F. Böhle, A. Jullien, J. P. Rousseau, T. Lefrou, D. Douillet, G. Iaquaniello, R. Lopez-Martens, A. Lifschitz, and J. Faure, Effect of the Laser Wave Front in a Laser-Plasma Accelerator, Phys. Rev. X 5, 031012 (2015); J. Ferri, X. Davoine, S. Fourmaux, J. Kieffer, S. Corde, K. T. Phuoc, and A. Lifschitz, Effect of experimental laser imperfections on laser wakefield acceleration and betatron source, Sci. Rep. 6, 27846 (2016).

[5] A. Popp, J. Vieira, J. Osterhoff, Z. Major, R. Hörlein, M. Fuchs, R. Weingartner, T. P. Rowlands-Rees, M. Marti, R. A. Fonseca, S. F. Martins, L. O. Silva, S. M. Hooker, F. Krausz, F. Grüner, and S. Karsch, All-Optical Steering of Laser-Wakefield-Accelerated Electron Beams, Phys. Rev. Lett. 105, 215001 (2010).

[6] M. Schnell et al., Optical control of hard X-ray polarization by electron injection in a laser wakefield accelerator, Nat. Commun. 4, 2421 (2013).

[7] S. Fourmaux et al., Single shot phase contrast imaging using laser-produced Betatron x-ray beams, Opt. Lett. 36, 2426 (2011).

[8] W. Leemans and E. Esarey, Laser-driven plasma-wave electron accelerators, Phys. Today 62, No. 3, 44 (2009).

[9] H.-P. Schlenvoigt et al., A compact synchrotron radiation source driven by a laser-plasma wakefield accelerator, Nat. Phys. 4, 130 (2008); S. Kneip et al., X-ray phase contrast imaging of biological specimens with femtosecond pulses of betatron radiation from a compact laser plasma wakefield accelerator, Appl. Phys. Lett. 99, 093701 (2011).

[10] V. Malka, J. Faure, Y. A. Gauduel, E. Lefebvre, A. Rousse, and K. T. Phuoc, Principles and applications of compact laser-plasma accelerators, Nat. Phys. 4, 447 (2008).

[11] J. Cole, K. Behm, E. Gerstmayr, T. Blackburn, J. Wood, C. Baird, M. J. Duff, C. Harvey, A. Ilderton, A. Joglekar et al., Experimental Evidence of Radiation Reaction in the Collision of a High-Intensity Laser Pulse with a LaserWakefield Accelerated Electron Beam, Phys. Rev. X 8, 011020 (2018).

[12] K. Poder, M. Tamburini, G. Sarri, A. Di Piazza, S. Kuschel, C. Baird, K. Behm, S. Bohlen, J. Cole, D. Corvan et al., Experimental Signatures of the Quantum Nature of Radiation Reaction in the Field of an Ultraintense Laser, Phys. Rev. X 8, 031004 (2018).

[13] C. B. Schroeder, E. Esarey, C. G. R. Geddes, C. Benedetti, and W. P. Leemans, Physics considerations for laser- plasma linear colliders, Phys. Rev. ST Accel. Beams 13, 101301 (2010).

[14] S. Steinke et al., Multistage coupling of independent laserplasma accelerators, Nature (London) 530, 190 (2016).

[15] J.-L. Vay et al., Nucl. Instrum. Methods Phys. Res., Sect. A 909, 476 (2018).

[16] J.-L. Vay, D. Grote, R. Cohen, and A. Friedman, Novel methods in the particle-in-cell accelerator code-framework warp, Comput. Sci. Discovery 5, 014019 (2012).

[17] S. Akturk, X. Gu, E. Zeek, and R. Trebino, Pulse-front tilt caused by spatial and temporal chirp, Opt. Express 12, 4399 (2004).

[18] J. B. Cole, A high-accuracy realization of the Yee algorithm using non-standard finite differences, IEEE Trans. Microwave Theory Tech. 45, 991 (1997); Highaccuracy Yee algorithm based on nonstandard finite differences: New developments and verifications, IEEE Trans. Antennas Propag. 50, 1185 (2002); M. Karkkainen, E. Gjonaj, T. Lau, and T. Weiland, Low-dispersion wakefield calculation tools, in Proceedings of the International Computational Accelerator Physics Conference, Chamonix, France (CERN, Geneva, Switzerland, 2006), pp. 35-40.

[19] B. M. Cowan, D. L. Bruhwiler, E. Cormier-Michel, E. Esarey, C. G. Geddes, P. Messmer, and K. M. Paul, Characteristics of an envelope model for laser-plasma accelerator simulation, J. Comput. Phys. 230, 61 (2011).

[20] B. B. Godfrey and J.-L. Vay, Suppressing the numerical cherenkov instability in FDTD PIC codes, J. Comput. Phys. 267, 1 (2014).

[21] J.-L. Vay, Noninvariance of Space- and Time-Scale Ranges under a Lorentz Transformation and the Implications for the Study of Relativistic Interactions, Phys. Rev. Lett. 98, 130405 (2007).

[22] J.-L. Vay, C. Geddes, E. Esarey, C. Schroeder, W. Leemans, E. Cormier-Michel, and D. Grote, Modeling of $10 \mathrm{GeV}-1 \mathrm{TeV}$ laser-plasma accelerators using Lorentz boosted simulations, Phys. Plasmas 18, 123103 (2011).

[23] L. M. Gorbunov and V. I. Kirsanov, Excitation of plasma waves by an electromagnetic wave packet, Zh. Eksp. Teor. Fiz. 93, 509 (1987) [Sov. Phys. JETP 66, 40 (1987)].

[24] D. E. Mittelberger, M. Thévenet, K. Nakamura, A. J. Gonsalves, C. Benedetti, S. Steinke, J. Daniels, R. Lehe, C. B. Schroeder, J.-L. Vay, E. Esarey, and W. P. Leemans Laser and Electron Deflection from Lateral Asymmetry in Laser Plasma Accelerators (to be published).

[25] K. Nakamura, H. S. Mao, A. J. Gonsalves, H. Vincenti, D. E. Mittelberger, J. Daniels, A. Magana, C. Toth, and W. P. Leemans, Diagnostics, control and performance parameters for the BELLA high repetition rate petawatt class laser, IEEE J. Quantum Electron. 53, 1 (2017).

[26] X. Xu, J. Hua, Y. Wu, C. Zhang, F. Li, Y. Wan, C.-H. Pai, W. Lu, W. An, P. Yu et al., Physics of Phase Space Matching for Staging Plasma and Traditional Accelerator Components Using Longitudinally Tailored Plasma Profiles, Phys. Rev. Lett. 116, 124801 (2016).

[27] I. Dornmair, K. Floettmann, and A. R. Maier, Emittance conservation by tailored focusing profiles in a plasma accelerator, Phys. Rev. ST Accel. Beams 18, 041302 (2015). 